\title{
"I Don't Mind Doing What It Takes to be Safe." Patient Perspectives of Urine Drug Testing for Pain
}

\author{
Jessica Wyse, $P h D^{1,2}$ (1) , Akeesha Simmons, $B A^{2}$, Banu Ramachandran, $J^{3}$, \\ Steven K. Dobscha, MD ${ }^{2,4}$, and Benjamin J. Morasco, $P h D^{2,4}$
}

${ }^{1}$ School of Public Health, Oregon Health \& Science University, Portland, OR, USA; ${ }^{2}$ Center to Improve Veteran Involvement in Care, VA Portland Health Care System, Portland, OR, USA; ${ }^{3}$ School of Medicine, Oregon Health \& Science University, Portland, OR, USA; ${ }^{4}$ Department of Psychiatry , Oregon Health \& Science University, Portland, OR, USA.

\begin{abstract}
KEY WORDS: urine drug testing; long-term opioid therapy; chronic pain; primary care.

$\mathrm{J}$ Gen Intern Med 36(1):243-4

DOI: $10.1007 / \mathrm{s} 11606-020-05688-3$

(c) Society of General Internal Medicine (This is a U.S. government work and not under copyright protection in the U.S.; foreign copyright protection may apply) 2020
\end{abstract}

\section{INTRODUCTION}

Urine drug testing (UDT) is a standard recommendation for those prescribed long-term opioid therapy (LTOT) for pain, ${ }^{1}$ but remains underutilized. ${ }^{2}$ Clinician fears regarding negative patient perceptions have been identified as a barrier to conducting UDT; ${ }^{3}$ however, little is known about patient perspectives of UDT. This study sought to uncover patients' beliefs regarding UDT and its implications for the patient-clinician relationship.

\section{METHODS}

These data are a secondary analysis from a larger, IRBapproved study. ${ }^{4}$ Informed consent was obtained from all participants. Eligible participants received care at VA Portland Health Care System and were prescribed opioids for $90+$ days for chronic musculoskeletal pain. Participants completed structured visits every 6 months for 24 months from 2013 to 2017. UDTs were completed as part of clinical care, not study procedures. At the final study visit, survey questions asked participants to choose between fixed response categories; open-ended questions probed experiences and perspectives regarding UDT. Qualitative data were double-coded by two research team members and categorized into themes. Representative quotes are presented in the text and Table 1.

Received January 3, 2020

Revised January 3, 2020

Accepted January 27, 2020

Published online February 10, 2020

\section{RESULTS}

The questionnaire was completed by 145 participants. Participants were primarily white (88\%), and male (90\%) with average age $62(\mathrm{SD}=10.1$, range $=34-87)$.

Overall, participants were indifferent to UDT and felt it did not impact the patient-provider relationship. This was captured in comments like, "It's just like when they ask you to do a lab test, it's no big deal." The ubiquity of UDT (e.g., in the military or workplace) had normalized testing, and UDT was generally viewed as clinicians "just doing their job."

Participants understood UDT as a sensible and necessary component of clinical care. As one participant stated, "They make perfect sense. If they [clinicians] are going to supply them [opioid medications], then obviously there should be some sort of monitoring." For some, guidelines in the opioid treatment agreement primed their expectations for UDT; others couched their beliefs within the context of the opioid crisis, “... they're absolutely necessary. I'm just one guy and I know I take it but if you're a doctor I can't imagine what stories they get." These participants understood that their own clinical care was embedded within a larger landscape, and thus UDT did not reflect clinicians' beliefs about them personally. UDT was also viewed as an important means of protecting health and safety. As one participant described, "I don't mind doing what it takes to be safe." Participants recognized that opioid medications were "powerful" and "potentially dangerous," and thus UDT “... might end up saving somebody's life in the end." Further, as one participant described, "It actually made our relationship even stronger. I can deal with anything that is required ... as long as you ... tell me the truth about what it really is." For some participants, UDT opened lines of communication, eliminated clinicians' doubts about their behavior, and indicated concern for their well-being.

A small minority expressed dissatisfaction with UDT and described it as worsening the relationship with their clinician. For these participants, UDT evidenced a lack of trust, "You think...well they don't trust you. But I realize it's not personal." Others did take UDT personally describing it as "invasive," and "a violation of privacy."

These findings are mirrored in the survey results (Table 2), where nearly $90 \%$ of participants recognized the benefits of 
Table 1 Patient Perspectives of Urine Drug Testing and Impacts on the Patient-Clinician Relationship

\begin{tabular}{ll}
\hline \hline Theme & Illustrative quote \\
\hline
\end{tabular}

Patient perceptions of the value and purpose of UDT: "How do you feel about urine drug tests in general?"

Necessary and sensible "They make perfect sense. If they [clinicians] are going to supply them [opioid medications], then obviously there should be some sort of monitoring."

"There are so many people that abuse this stuff...I've seen it with my own eyes."

"I had a roommate who passed away from opioid overdose."

Protective of health and "I don't mind doing what it takes to be safety safe."

[Opioid medications are] "powerful" [and] "potentially dangerous," [and UDT] "...might end up saving somebody's life in the end."

Indifferent and expected "They don't bother me at all," and "It's just like when they ask you to do a lab test, it's no big deal."

"I don't mind them. I mean they do that on [the] job, random drug tests so it makes sense for medical stuff."

Insulting, invasive, and "I understand why it has to be done. I'm never happy about it. You think, oh, well they don't trust you. But I realize it's not personal."

"You're a suspect. You feel interrogated." Urine drug testing and the patient-clinician relationship: "How did the urine drug test affect your relationship with your provider, if at all?"

Relationship unaffected "It didn't."

"Not at all."

[Doctors are] "just doing their job."

Strengthened trust and communication

"It actually made our relationship even stronger. Because we got to sit down and actually talk about why things are the way they are and why they have to be this way...I can deal with anything that is required with me as long as you sit down and tell me the truth about what it really is."

"It proved to him in black and white what I'm doing," [and thereby] "creat[ed] a level of trust."

Fostered distress and distrust
[UDT is a] "way of having more control."

[My doctor was] "upset about the alcohol."

[UDT positive for marijuana] "would not go over well with the VA. Most doctors do not agree with marijuana."
UDT or were indifferent to them and described no change in their relationship as a result of UDT.

\section{DISCUSSION}

Routine UDT of patients prescribed LTOT remains underutilized, in part due to clinician concerns about patient perceptions. ${ }^{3}$ Our results suggest that clinicians' fears may be misplaced. Patients were largely indifferent to UDT and understood it to be a standard aspect of care, motivated by a desire to protect their health. The broader context of the opioid crisis framed patient understanding of the need for UDT, as many were aware of the consequences of opioid misuse and abuse, which UDTs are designed to identify. To assuage
Table 2 Patient Perspectives of Urine Drug Testing and Impacts on the Patient-Clinician Relationship $(n=145)$

\begin{tabular}{ll}
\hline \hline & $\begin{array}{l}\text { Participants } \\
(\boldsymbol{\%}(\boldsymbol{n}))\end{array}$ \\
\hline How do you feel about urine drug tests in general? & \\
$\quad$ I dislike them & $9.0 \%(13)$ \\
I am indifferent & $17.2 \%(25)$ \\
I see the benefit and don't mind taking urine drug tests & $72.4 \%(105)$ \\
Other/missing & $1.4 \%(2)$ \\
How did taking the urine drug test affect your & \\
relationship with your provider, if at all?* & \\
No change & $88.8 \%(127)$ \\
Improved relationship & $4.9 \%(7)$ \\
Damaged the relationship & $5.6 \%(8)$ \\
Other & $0.7 \%(1)$ \\
\hline
\end{tabular}

*Two participants were missing data on this question

concerns among the small minority of patients with negative perceptions of UDT, clinicians may wish to emphasize that the decision to utilize UDT is not personal, but a standard aspect of clinical care generally driven by desires to improve safety. ${ }^{5}$ Overall, these data suggest that fears of negative patient perceptions should not deter clinicians from conducting UDTs.

Acknowledgments: We would like to thank Stephanie Hyde for research assistance with this project.

Corresponding Author: Jessica Wyse, PhD; Center to Improve Veteran Involvement in Care VA Portland Health Care System, Portland, OR, USA (e-mail: wyse@ohsu.edu).

\section{Compliance with Ethical Standards:}

The content of this manuscript is solely the responsibility of the authors and does not necessarily represent the official views of the Department of Veterans Affairs, the National Institute on Drug Abuse or the Agency for Healthcare Research and Quality.

Conflict of Interest: Research reported in this manuscript was supported by grant 034083 from the National Institute on Drug Abuse of the National Institutes of Health. The work was also supported by resources from the VA Health Services Research and Developmentfunded Center to Improve Veteran Involvement in Care at the VA Portland Health Care System (CIN 13-404). Dr. Wyse's time was also supported by grant number K12HSO26370 from the Agency for Healthcare Research and Quality. No author reports having any potential conflict of interest with this study.

\section{REFERENCES}

1. Dowell D, Haegerich TM, Chou R. CDC guideline for prescribing opioids for chronic pain-United States, 2016. Jama. 2016;315(15):1624-1645.

2. Starrels JL, Becker WC, Weiner MG, Li X, Heo M, Turner BJ. Low use of opioid risk reduction strategies in primary care even for high risk patients with chronic pain. J Gen Intern Med 2011;26(9):958-964.

3. Krebs EE, Bergman AA, Coffing JM, Campbell SR, Frankel RM, Matthias MS. Barriers to guideline-concordant opioid management in primary care-a qualitative study. J Pain 2014;15(11):1148-1155.

4. Morasco BJ, Smith N, Dobscha SK, Deyo RA, Hyde S, Yarborough BJH. Outcomes of prescription opioid dose escalation for chronic pain: Results from a prospective cohort study. Pain (in press)

5. Wyse JJ, Ganzini L, Dobscha SK, Krebs EE, Morasco BJ. Setting Expectations, Following Orders, Safety, and Standardization: Clinicians'

Publisher's Note Springer Nature remains neutral with regard to jurisdictional claims in published maps and institutional affiliations. 